\title{
Review Article \\ Networked Control Systems: The Communication Basics and Control Methodologies
}

\author{
Yun-Bo Zhao, ${ }^{1}$ Xi-Ming Sun, ${ }^{2}$ Jinhui Zhang, ${ }^{3}$ and Peng Shi ${ }^{4,5}$ \\ ${ }^{1}$ Department of Automation, Zhejiang University of Technology, Hangzhou 310023, China \\ ${ }^{2}$ Research Center of Information and Control, Dalian University of Technology, Dalian 116024, China \\ ${ }^{3}$ College of Information Science and Technology, Beijing University of Chemical Technology, Beijing 100029, China \\ ${ }^{4}$ College of Automation, Harbin Engineering University, Harbin, Heilongjiang 150001, China \\ ${ }^{5}$ School of Electrical and Electronic Engineering, University of Adelaide, Adelaide, SA 5005, Australia
}

Correspondence should be addressed to Yun-Bo Zhao; yunbo.zhao@imperial.ac.uk

Received 11 December 2014; Accepted 23 February 2015

Academic Editor: Bo Shen

Copyright (c) 2015 Yun-Bo Zhao et al. This is an open access article distributed under the Creative Commons Attribution License, which permits unrestricted use, distribution, and reproduction in any medium, provided the original work is properly cited.

As an emerging research field, networked control systems have shown the increasing importance and attracted more and more attention in the recent years. The integration of control and communication in networked control systems has made the design and analysis of such systems a great theoretical challenge for conventional control theory. Such an integration also makes the implementation of networked control systems a necessary intermediate step towards the final convergence of control, communication, and computation. We here introduce the basics of networked control systems and then describe the state-of-theart research in this field. We hope such a brief tutorial can be useful to inspire further development of networked control systems in both theory and potential applications.

\section{Introduction}

"Networked control systems" (NCSs) are the name of a general class of control systems where "the control loop is closed via a serial communication network" [1]. Interest in such a system configuration can date back to as early as 1980s, when the so-called "Integrated Communication and Control Networks" attracted much attention from the control community [2]. From that time on, other aliases, such as "Network-Based Control Systems" and "Control over (through) Networks," have also been used to describe similar, if not the same, system configuration as NCSs but are seldom used today.

As indicated by its name, the most distinct feature of NCSs is the use of communication networks in the control loop $[3,4]$. Earlier days have witnessed the use of the controloriented communication networks, such as the Control Area Network and DeviceNet, as the first choice of the communication networks in NCSs; the fast development of the communication technology as well as the increasing needs of large scale systems has now made the Internet an overwhelmingly attracting alternative. The Internet offers us the capability of building a large control system at much lower cost and easier maintenances, with also more flexible reconfiguration. Built on such fundamental theoretical advances in NCSs, we have seen various innovations, such as the smart home, smart transportation, remote surgery, and Internet of Things, in recent years [5-8].

The advantages brought by NCSs however do not come at no cost. A fundamental basis of conventional control systems is that the data exchanges among the control components are lossless. In NCSs, the data have to be transmitted through the communication network, and the nature of the Internet and other variations of data networks means that perfect data exchanges among the control components are essentially unavailable. The imperfect data translation in NCSs thus introduces the so-called communication constraints to the control system, which include, for example, the network-induced delay (the delays occurred in transmitting the sensing and control data), 


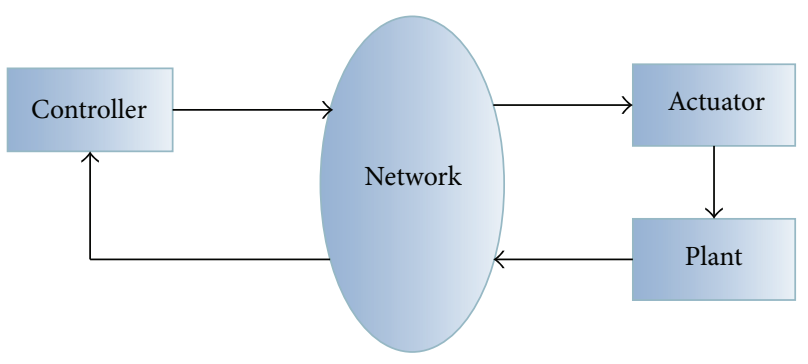

FIGURE 1: Networked control systems in the direct structure.

the data packet dropout (the data packet may be missing during transmission), and the time synchronization issue (different control components may work on different clocks) [9]. These communication constraints can greatly degrade the system performance or even destabilize the system at certain conditions, while simple extensions of conventional control approaches cannot be obtained directly in a networked control environment [10-16]. These difficulties thus pose great challenges for the control and communication communities and considerable works have been done for a better understanding and design of such systems at the boundary of control theory and communication technology [17-19].

Here, we provide a brief tutorial on NCSs. This consists of two parts. We first give an extensive introduction of the communication networks in NCSs, including its basic characteristics and more importantly its interactions with the control system. Note that we focus on data networks such as the Internet but not the control-oriented networks, simply because of the increasing use and more complicated communication features of the former. We then survey the state-of-the-art research on NCSs, from mainly the control perspective with also an emphasis on the codesign approach which integrates both control and communication. This tutorial is not necessarily thorough or comprehensive. Rather, our main purpose is to introduce to the new researchers the basics of NCSs. By attracting more and more young researchers to this field, we believe that the glorious future that NCSs have promised will become true very soon.

For simplicity in this tutorial, we focus on a simpler structure of NCSs. In fact, from a general perspective of system structure, NCSs may contain two different structures [20]: the "direct structure" (Figure 1) and the "hierarchical structure" (Figure 2). The latter is different from the former as a local controller is present and the communication network is used to close the loop between the main controller and the local system. This structural distinction may have some theoretical as well as practical values; the latter, however, may be regarded as a hierarchical combination of the direct structured NCS and a conventional local control system and therefore it is not absolutely necessary to investigate the hierarchical structure as a brand new type of NCSs. In fact, most available works on NCSs to date have focused on the direct structure, which is also the main focus of this brief tutorial.

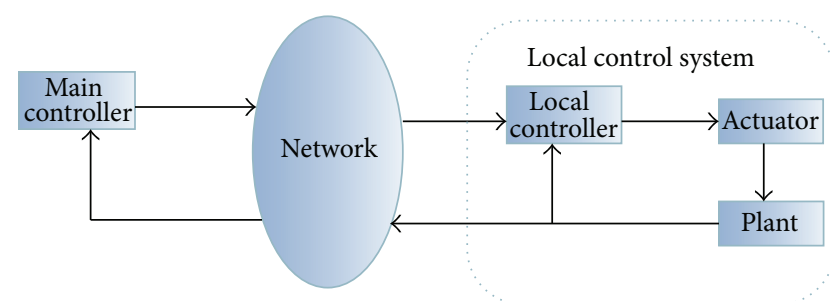

FIGURE 2: Networked control systems in the hierarchical structure.

\section{The Basics of Networked Control Systems}

This section introduces the basics of NCSs, where an emphasis is made on the differences between NCSs and conventional control systems, that is, the distinct and unique characteristics of NCSs that are brought by the inserted communication network.

2.1. Network Topology. In the presence of the communication network in the NCSs, the conventional control components include the sensor, the controller, and the actuator work as network nodes from the perspective of network topology. From this perspective, two issues need to be addressed as follows.

2.1.1. Time-Synchronization. The control components need to be time synchronized to act properly. This is a fundamental basis of conventional control systems but is usually missing in NCSs due to the use of the distributed communication networks [21]. Under certain conditions, time-synchronization in NCSs may not be a necessary condition if the networkinduced delay in the backward channel is not required for the calculation of the control signals and/or the networkinduced delay in the forward channel is not required for the implementation of the control actions. In some other cases, as discussed in $[22,23]$, time-synchronization together with the use of time stamps in NCSs can offer an advantage over conventional time delay systems since the backward channel delay is known by the controller and the forward channel delay (round trip delay as well) is known by the actuator. This advantage can then be used to derive a better control structure for NCSs as done in $[22,24]$.

2.1.2. Drive Mechanism. The sensor and the actuator can be driven either by time or event. The difference between the two drive mechanisms lies in the trigger method that initiates the control components. For the time-driven mechanism, the control components are trigged to work at regular intervals, while for the event-driven mechanism the control components are only trigged by predefined "events." From a broad perspective, time-driven mechanism can be regarded as a special case of event-driven mechanism, when the trigger events for the latter are chosen as the time. Therefore, it is no wonder why the event-driven mechanisms are more sophisticated and may require ancillary devices to work.

The sensor is usually time driven, while the controller and the actuator can either be time driven or event driven. For 


\begin{tabular}{|l|l|l|l|l|l|}
\hline Header & NCS & System 1 & System 2 & System... & Trailer \\
\hline & \multicolumn{5}{|c|}{ Payload } \\
\hline
\end{tabular}

FIGURE 3: The typical data packet structure where NCS is sharing the data packet with other applications.

more information on the drive mechanism for the control components, the reader is referred to [25] and the references therein. It is worth mentioning though that, with different drive mechanisms, different system models for NCSs are obtained and event-driven control components generally lead to a better system performance.

2.2. Packet-Based Data Transmission. The data in NCSs is encoded in the data packets and then transmitted through the communication network. A typical data packet is shown in Figure 3. Packet-based transmission is one of the most important characteristics of NCSs which distinguishes it from conventional control systems [26-28]. This characteristic can mean that the perfect data transmission as assumed in conventional control systems is absent in NCSs, posing the most challenging aspect in NCSs. The communication constraints caused by the packet-based transmission in NCSs include the network-induced delay, data packet dropout, and data packet disorder, which are detailed in what follows.

2.2.1. Network-Induced Delay. The transmission time for the data packets introduces network-induced delays to NCSs, which are well known to degrade the performance of the control systems.

There are two types of network-induced delays according to where they occur.

(i) $\tau_{\mathrm{sc}}$ is network-induced delay from the sensor to the controller, that is, backward channel delay.

(ii) $\tau_{\mathrm{ca}}$ is network-induced delay from the controller to the actuator, that is, forward channel delay.

The two types of network-induced delays may have different characteristics [29]. In most cases, however, these delays are not treated separately and only the round trip delay is of interest [4, 30-32].

According to the types of the communication networks being used in NCSs, the characteristics of the networkinduced delay vary as follows $[20,33,34]$.

(i) Cyclic service networks (e.g., Toking-Ring and Toking-Bus) are bounded delays which can be regarded as constant for most occasions.

(ii) Random access networks (e.g., Ethernet and CAN) are random and unbounded delays.

(iii) Priority order networks (e.g., DeviceNet) are bounded delays for the data packets with higher priority and unbounded delays for those with lower priority.

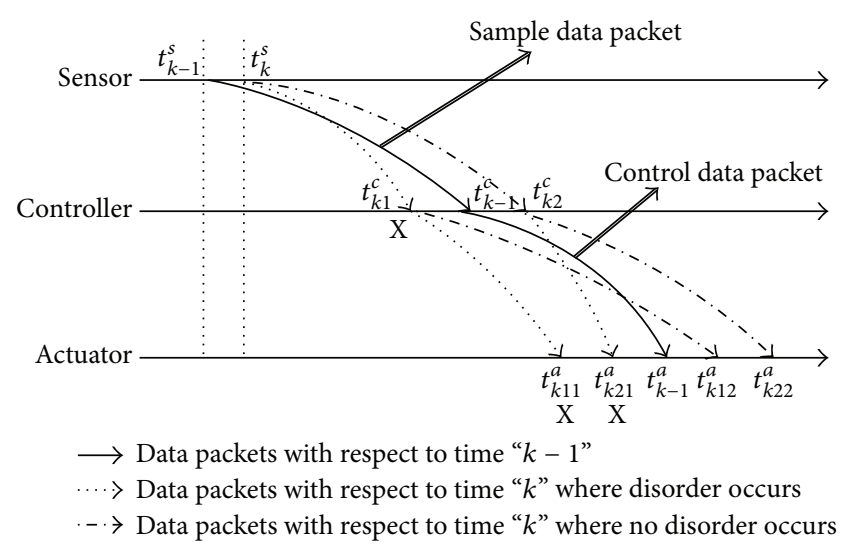

FIGURE 4: Data packet disorder in NCSs.

Network-induced delay is one of the most important characteristics of NCSs which has been widely addressed in the literature to date; see, for example, [4, 30, 32, 35-48].

2.2.2. Data Packet Dropout. Data transmission error in communication networks is inevitable, which in the case of NCSs then produces a situation called "data packet dropout." Data packet dropout can occur either in the backward or forward channel, and it makes either the sensing data or the control signals unavailable to NCSs, thus significantly degrading the performance of NCSs.

In communication networks, two different strategies are applied when a data packet is lost, that is, either to send the packet again or simply discard it. Using the terms from communication networks, these two strategies are called transmission control protocol (TCP) and user datagram protocol (UDP), respectively [21]. It is readily seen that, with TCP, all the data packets will be received successfully, although it may take a considerably long time for some data packets, while, with UDP, some data packets will be lost forever.

As far as NCSs are concerned, UDP is used in most applications due to the real-time requirement and the robustness of control systems. As a result, the effect of data packet dropout in NCSs has to be explicitly considered, as done in, for example, [49-54].

2.2.3. Data Packet Disorder. In most communication networks, different data packets suffer different delays, which then produces a situation where a data packet sent earlier may arrive at the destination later or vice versa; see Figure 4 . This phenomenon is referred to as data packet disorder. The existence of data packet disorder can mean that a newly arrived control signal in NCSs may not be the latest, which never occurs in conventional control systems. The control performance will be inevitably degraded if the control algorithm has not taken explicit consideration of the disordered data. Some preliminary works have been done, usually using an active compensation scheme [55-57]. 


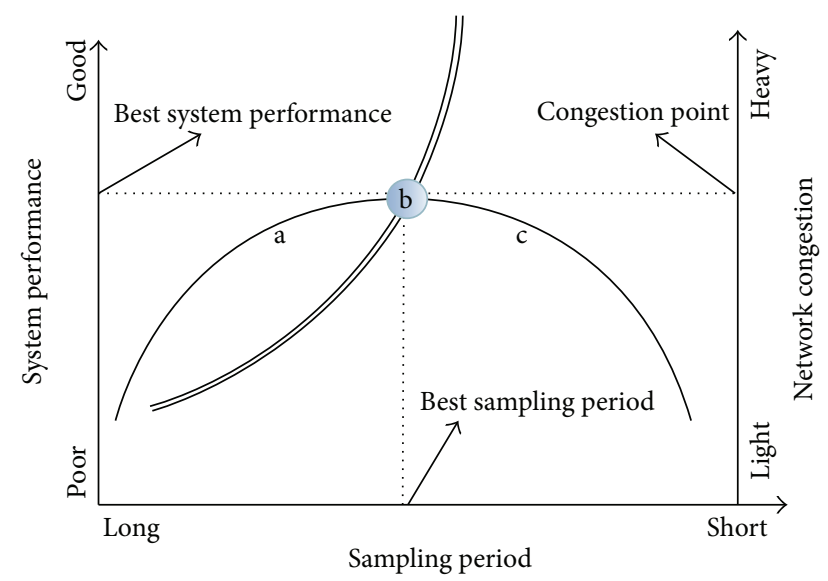

- System performance

= Network congestion

FIGURE 5: Relationship between the sampling period, network loads, and system performance in NCSs.

2.2.4. Single and Multipacket. When the sensing data and the control signals are sent via data packets of the network, another situation occurs: in a case where, for example, multiple sensors are used and distributed geographically in NCSs and thus they send their sensing data separately to the controller over the network, the controller may have to wait for the arrival of all the sensing data packets before it is able to calculate the control actions, and if only one sensing data packet is lost, all the other sensing data packets have to be discarded due to incompleteness. We call this situation the "multipacket" transmission of the data in NCSs.

Another situation in NCSs is where the sensing data or the control signals of multiple steps are sent via a single data packet over the network, since the packet size used in NCSs can be very large compared with the data size required to encode a single step of sensing data or control signal. This "single packet" transmission of the data in NCSs is the fundamental basis of the so-called packet-based control approach [24].

2.3. Limited Network Resources. The limitation of the network resources in NCSs is primarily caused by the limited bandwidth of the communication network, which results in the following three situations in NCSs that are distinct from conventional control systems.

2.3.1. Sampling Period, Network Loads, and System Performance. NCSs are a special class of sampled data systems due to the digital transmission of the data in communication networks. However, in NCSs, the limited bandwidth of the network produces a situation where, a smaller sampling period may not result in a better system performance which is normally true for sampled data systems [58].

This situation happens because, with a too small sampling period, too much sensing data will be produced, thus overloading the network and causing congestion, which will result

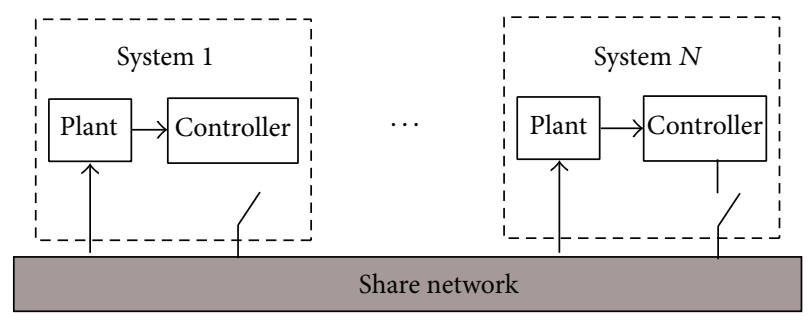

FIGURE 6: Multiple NCSs can share the communication network.

in more data packet dropouts and longer delays, and then degrading the system performance. The relationship between the sampling period, network loads, and system performance in NCSs is illustrated in Figure 5. For example, when the sampling period decreases from the value corresponding to points "a" to "b," the system performance is getting better as in conventional sampled data systems since the network congestion does not appear until point " $b$ "; however, the system performance is likely to deteriorate due to the network congestion when the sampling period is getting even smaller from the value corresponding to points " $b$ " to "c." Therefore, the relationship shown in Figure 5 implies that there is a trade-off between the period of sampling of the plant data and the system performance in NCSs; that is, in NCSs, an optimal sampling period exists which offers the best system performance (point "b" in Figure 5).

2.3.2. Quantization. Due to the use of data networks with limited bandwidth, signal quantization is inevitable in NCSs, which has a significant impact on the system performance. Quantization in the meantime is also a potential method to reduce the bandwidth usage which enables it to be an effective tool to avoid the network congestion in NCSs and thus improve the system performance of NCSs. For more information on the quantization effects in NCSs, the reader is referred to [59-64] and the references therein.

2.3.3. Network Access Constraint and Scheduling. As shown in Figure 3, an NCS may use only part of the payload and share the data packet with other applications. In particular, in Figure 6, the other applications can also be NCSs, meaning that multiple NCSs share the same communication network. In such a case, the limited bandwidth of the network means that subsystems may not be able to access the network resources at all times due to resource competition. A scheduling algorithm is therefore needed to schedule the timeline of when and how long a specific subsystem can occupy the network resource. At the same time, under the satisfactory control performance constraint, the less bandwidth an NCS uses, the better it does to other applications.

\section{The Research on Networked Control Systems}

In this section, we briefly survey the state-of-the-art research on NCSs. This consists of two parts, categorized according 
to the methodologies used in these research; that is, the first category is dominated by the use of the control theory, while the second one adopts a codesign strategy by combining control and communication together.

3.1. Control-Centred Research on NCSs. Since the renewed interest in NCSs [1], the research on NCSs has been primarily done within the control theory community [20].

From the control theory community, one is concerned with the theoretical analysis of the control performance of NCSs where the network in NCSs is modeled by predetermined parameters to the control system. In this type of research, the communication characteristics of NCSs, for example, the network-induced delay, can be formulated and incorporated into the system as some parameters, thereby yielding a conventional control system for further analysis and design. This type of research simplifies the modeling and analysis of NCSs, enabling all existing control methods to be readily applied to NCSs. Hence, such a research strategy has been dominating the research field for a significant period $[20,65]$.

Since the communication characteristics are assumed to be given parameters, the design of NCSs then faces great conservativeness. Most works can only focus on the extension of existing control approaches to NCSs without full use of the communication characteristics of NCSs. This then ignores the possibility of optimizing the system performance by making efficient use of the network characteristics [66-69].

The conventional control approaches and theories that have been applied to NCSs are briefly surveyed as follows.

(i) Time Delay Systems. As far as the network-induced delay is concerned, it is natural to model NCSs as a special class of time delay systems. This research method covers a vast range of research on NCSs; see, for example, [39, 41, 70-77] and the survey in $[4,20,78]$.

An interesting issue here is to determine the maximum allowable delay bound (MADB) of NCSs, which is the upper bound of the transfer interval that ensures the stability or other performance objectives of NCSs [79]. The determination of MADB is important in theory and can also play a guiding role for practical applications. One can refer to the survey paper in [36] for more information on this issue.

(ii) Stochastic Control. As mentioned above, the communication constraints in NCSs are stochastic in nature, thus enabling the application of conventional stochastic control approaches to NCSs. An early study can be found in [29], where the characteristics of the network-induced delay were explicitly formulated and preliminary stochastic stability criteria of NCSs were obtained; [35] extended the work in [2] to a stochastic optimal control framework and gave the stochastic optimal state feedback and output feedback controllers, respectively; in [42], the sufficient and necessary conditions of the stochastic stability of NCSs were obtained based on the Markov jump system framework. For further information, the reader is referred to the survey in [9]. (iii) Optimal Control. As a very successful idea both in theory and practical applications, optimal control has also found its position in NCSs. Undoubtedly, conventional optimal control approaches can be used in the networked control environment to design the controller for NCSs; see, for example, $[48,50,51,80-82]$; and, as a special class of optimal control approaches, model predictive control (MPC or receding horizon control (RHC)) seems to be more suitable for the networked control environment and "a major extension required to apply model predictive control in networked environments would be the distributed solution of the underlying optimization problem" [27]. Examples of the application of MPC to NCSs can be seen, for example, in $[23,44,83-86]$.

(iv) Switched System Theory. Another important tool in the study on NCSs is switched system theory, which is typically used by modelling different network conditions in NCSs as different system modes. This approach can readily deal with network-induced delay as well as data packet dropout in NCSs, and the limitation of the approach is caused mainly by how well we understand the properties of the changes of the network conditions, which is generally difficult. For the research in this area, the reader is referred to [72, 75, 87-93] and the references therein.

3.2. Codesign for NCSs. As has been pointed out earlier, it is the communication network which replaces the direct connections among the control components in conventional control systems that makes NCSs distinct. Therefore, the so-called codesign approach to NCSs, an approach that integrates both control and communication, has been an emerging trend in recent years. The communication constraints are no longer assumed as predetermined parameters but act as designable factors, and by the efficient use of these factors a better performance can be expected [23, 26, 67, 68, $85,94-97]$. We give two examples of the codesign approach to NCSs.

(i) Packet-Based Control Approach. As discussed in Section 2.2, the packet-based transmission is one of the most distinct characteristics of NCSs. This characteristic can be used to derive a codesign control structure for NCSs, called the packet-based control framework, as done in [23, 24, 98-100]. The packet-based control approach has its origin in $[44,97]$, where, with the use of generalized predictive control method, the packet-based structure of the data transmission was efficiently used to actively compensate for the communication constraints in NCSs.

(ii) Control and Scheduling Codesign. In NCSs, a situation may occur where multiple control components share a network with limited bandwidth. In such a situation, network resource scheduling among the control components is necessary; see Section 2.3.3. As far as the scheduling algorithms are concerned, [1] proposed a dynamic scheduling algorithm called "try-once-discard" (TOD) which allocates the network resources in a way that the node with the greatest error in the last reported period has access to the network resource. 
Reference [101] proposed a Lyapunov uniformly globally asymptotically stable (UGAS) protocol based on TOD, which is further improved in [102]. In [103], the authors used the technique of "communication sequence" (see also [104]) to deal with the network access constraint for such a system configuration and modeled the subsystems as switched systems with two modes "open loop" and "closed loop" which switch according to whether the current subsystem has access to the medium or not. In [105], the authors considered a special case of the configuration shown in Figure 6 where the channel from controller to actuator is linked directly, and the rate monotonic scheduling algorithm is applied to schedule the transmissions of the sensing data of the subsystems.

\section{Conclusions}

Despite all the achievements that have been made for networked control systems in the past decades, more efforts are still needed in the future. Most of these ongoing researches adopt the codesign methodology, and the collaborations between the control and communication as well as computation communities are desirable.

These collaborations will then reveal the values of networked control systems in broader perspectives, by looking into its close relationship with other systems such as the Internet of Things, cyber-physical systems, and multiagent systems. All these together then bring us the promising future of the networked intelligent automation.

\section{Conflict of Interests}

The authors declare that there is no conflict of interests regarding the publication of this paper.

\section{Acknowledgments}

This work was supported in part by the National Natural Science Foundation of China under Grants 61304048, 61174058, and 61473024, the Australian Research Council under Grant DP140102180 and Grant LP140100471, and the 111 Project under Grant B12018.

\section{References}

[1] G. C. Walsh, O. Beldiman, and L. Bushnell, "Asymptotic behavior of networked control systems," in Proceedings of the IEEE International Conference on Control Applications, vol. 2, pp. 1448-1453, Kohala Coast, Hawaii, August 1999.

[2] Y. Halevi and A. Ray, "Integrated communication and control systems. Part 1. Analysis," Journal of Dynamic Systems, Measurement and Control, Transactions of the ASME, vol. 110, no. 4, pp. 367-373, 1988.

[3] R. A. Gupta and M.-Y. Chow, "Networked control system: overview and research trends," IEEE Transactions on Industrial Electronics, vol. 57, no. 7, pp. 2527-2535, 2010.

[4] J. P. Hespanha, P. Naghshtabrizi, and Y. Xu, "A survey of recent results in networked control systems," Proceedings of the IEEE, vol. 95, no. 1, pp. 138-172, 2007.
[5] V. C. Gungor and G. P. Hancke, "Industrial wireless sensor networks: challenges, design principles, and technical approaches," IEEE Transactions on Industrial Electronics, vol. 56, no. 10, pp. 4258-4265, 2009.

[6] M. Kranz, P. Holleis, and A. Schmidt, "Embedded interaction: interacting with the internet of things," IEEE Internet Computing, vol. 14, no. 2, pp. 46-53, 2010.

[7] F. Mattern and C. Floerkemeier, "From the internet of computers to the internet of things," in From Active Data Management to Event-Based Systems and More, K. Sachs, I. Petrov, and P. Guerrero, Eds., vol. 6462 of Lecture Notes in Computer Science, pp. 242-259, Springer, Berlin, Germany, 2010.

[8] J. Davis, T. Edgar, J. Porter, J. Bernaden, and M. Sarli, "Smart manufacturing, manufacturing intelligence and demanddynamic performance," Computers and Chemical Engineering, vol. 47, pp. 145-156, 2012.

[9] P. Antsaklis and J. Baillieul, "Special issue on technology of networked control systems," Proceedings of the IEEE, vol. 95, no. 1, pp. 5-8, 2007.

[10] M. S. Mahmoud and M. Sabih, "Experimental investigations for distributed networked control systems," IEEE Systems Journal, vol. 8, no. 3, pp. 717-725, 2014.

[11] M. Nagahara, D. E. Quevedo, and J. Ostergaard, "Sparse packetized predictive control for networked control over erasure channels," IEEE Transactions on Automatic Control, vol. 59, no. 7, pp. 1899-1905, 2014.

[12] B. Xue, N. Li, S. Li, and Q. Zhu, "Moving horizon scheduling for networked control systems with communication constraints," IEEE Transactions on Industrial Electronics, vol. 60, no. 8, pp. 3318-3327, 2013.

[13] R. Postoyan and D. Nesic, "A framework for the observer design for networked control systems," IEEE Transactions on Automatic Control, vol. 57, no. 5, pp. 1309-1314, 2012.

[14] J.-N. Li, Y.-J. Pan, H.-Y. Su, and C.-L. Wen, "Stochastic reliable control of a class of networked control systems with actuator faults and input saturation," International Journal of Control, Automation and Systems, vol. 12, no. 3, pp. 564-571, 2014.

[15] M. Pajic, S. Sundaram, G. J. Pappas, and R. Mangharam, "The wireless control network: a new approach for control over networks," IEEE Transactions on Automatic Control, vol. 56, no. 10, pp. 2305-2318, 2011.

[16] N. S. Mkondweni and R. Tzoneva, "LabNS2-co-simulation, co-emulation, and real-time control toolkit for investigation of network induced time delays and packet loss in networked control systems," International Journal of Innovative Computing, Information and Control, vol. 10, no. 1, pp. 1-18, 2014.

[17] K. Y. You and L. H. Xie, "Survey of recent progress in studying networked control systems," Acta Automatica Sinica, vol. 39, no. 2, pp. 101-118, 2013.

[18] L. Zhang, H. Gao, and O. Kaynak, "Network-induced constraints in networked control systems: a survey," IEEE Transactions on Industrial Informatics, vol. 9, no. 1, pp. 403-416, 2013.

[19] Y. Ge, L. Tian, and Z. Liu, "Survey on the stability of networked control systems," Journal of Control Theory and Applications, vol. 5, no. 4, pp. 374-379, 2007.

[20] Y. Tipsuwan and M.-Y. Chow, "Control methodologies in networked control systems," Control Engineering Practice, vol. 11, no. 10, pp. 1099-1111, 2003.

[21] W. Stallings, Data and Computer Communications, Prentice Hall, Englewood Cliffs, NJ, USA, 6th edition, 2000. 
[22] W. Zhang, M. S. Branicky, and S. M. Phillips, "Stability of networked control systems," IEEE Control Systems Magazine, vol. 21, no. 1, pp. 84-99, 2001.

[23] Y.-B. Zhao, G. P. Liu, and D. Rees, "Integrated predictive control and scheduling co-design for networked control systems," IET Control Theory \& Applications, vol. 2, no. 1, pp. 7-15, 2008.

[24] Y.-B. Zhao, G.-P. Liu, and D. Rees, "Design of a packetbased control framework for networked control systems," IEEE Transactions on Control Systems Technology, vol. 17, no. 4, pp. 859-865, 2009.

[25] W. Hu, G. Liu, and D. Rees, "Event-driven networked predictive control," IEEE Transactions on Industrial Electronics, vol. 54, no. 3, pp. 1603-1613, 2007.

[26] J. Baillieul and P. J. Antsaklis, "Control and communication challenges in networked real-time systems," Proceedings of the IEEE, vol. 95, no. 1, pp. 9-28, 2007.

[27] R. M. Murray, Ed., Control in an Information-Rich World: Report of the Panel on Future Directions in Control, Dynamics, and Systems, Society for Industrial and Applied Mathematics, 2003.

[28] R. M. Murray, K. J. Astrom, S. P. Boyd, R. W. Brockett, and G. Stein, "Future directions in control in an information-rich world," IEEE Control Systems Magazine, vol. 23, no. 2, pp. 2033, 2003.

[29] J. Nilsson, B. Bernhardsson, and B. Wittenmark, "Stochastic analysis and control of real-time systems with random time delays," Automatica, vol. 34, no. 1, pp. 57-64, 1998.

[30] L.-S. Hu, T. Bai, P. Shi, and Z. Wu, "Sampled-data control of networked linear control systems," Automatica, vol. 43, no. 5, pp. 903-911, 2007.

[31] Y. Fan, Z.-P. Jiang, and H. Zhang, "Network flow control under capacity constraints: a case study," Systems \& Control Letters, vol. 55, no. 8, pp. 681-688, 2006.

[32] F. Yang, Z. Wang, Y. S. Hung, and M. Gani, “ $H_{\infty}$ control for networked systems with random communication delays," IEEE Transactions on Automatic Control, vol. 51, no. 3, pp. 511-518, 2006.

[33] F.-L. Lian, Analysis, design, modeling, and control of networked control systems [Ph.D. dissertation], University of Michigan, Ann Arbor, Mich, USA, 2001.

[34] F.-L. Lian, J. R. Moyne, and D. M. Tilbury, "Performance evaluation of control networks: Ethernet, ControlNet, and DeviceNet," IEEE Control Systems Magazine, vol. 21, no. 1, pp. 66-83, 2001.

[35] S. Hu and Q. Zhu, "Stochastic optimal control and analysis of stability of networked control systems with long delay," Automatica, vol. 39, no. 11, pp. 1877-1884, 2003.

[36] D.-S. Kim, Y. S. Lee, W. H. Kwon, and H. S. Park, "Maximum allowable delay bounds of networked control systems," Control Engineering Practice, vol. 11, no. 11, pp. 1301-1313, 2003.

[37] F.-L. Lian, J. Moyne, and D. Tilbury, "Modelling and optimal controller design of networked control systems with multiple delays," International Journal of Control, vol. 76, no. 6, pp. 591606, 2003.

[38] L. A. Montestruque and P. J. Antsaklis, "On the model-based control of networked systems," Automatica, vol. 39, no. 10, pp. 1837-1843, 2003.

[39] D. Yue, Q.-L. Han, and C. Peng, "State feedback controller design of networked control systems," IEEE Transactions on Circuits and Systems II: Express Briefs, vol. 51, no. 11, pp. 640644, 2004.
[40] A. Sala, "Computer control under time-varying sampling period: an LMI gridding approach," Automatica, vol. 41, no. 12, pp. 2077-2082, 2005.

[41] D. Yue and Q.-L. Han, "Delayed feedback control of uncertain systems with time-varying input delay," Automatica, vol. 41, no. 2, pp. 233-240, 2005.

[42] L. Zhang, Y. Shi, T. Chen, and B. Huang, "A new method for stabilization of networked control systems with random delays," IEEE Transactions on Automatic Control, vol. 50, no. 8, pp. 11771181, 2005.

[43] X. Fan and M. Arcak, "Delay robustness of a class of nonlinear systems and applications to communication networks," IEEE Transactions on Automatic Control, vol. 51, no. 1, pp. 139-144, 2006

[44] G. P. Liu, J. X. Mu, D. Rees, and S. C. Chai, “Design and stability analysis of networked control systems with random communication time delay using the modified MPC," International Journal of Control, vol. 79, no. 4, pp. 288-297, 2006.

[45] X. Liu and J. Shen, "Stability theory of hybrid dynamical systems with time delay," IEEE Transactions on Automatic Control, vol. 51, no. 4, pp. 620-625, 2006.

[46] P. L. Tang and C. W. de Silva, "Compensation for transmission delays in an ethernet-based control network using variablehorizon predictive control," IEEE Transactions on Control Systems Technology, vol. 14, no. 4, pp. 707-718, 2006.

[47] M. García-Rivera and A. Barreiro, "Analysis of networked control systems with drops and variable delays," Automatica, vol. 43, no. 12, pp. 2054-2059, 2007.

[48] L. Schenato, B. Sinopoli, M. Franceschetti, K. Poolla, and S. S. Sastry, "Foundations of control and estimation over lossy networks," Proceedings of the IEEE, vol. 95, no. 1, pp. 163-187, 2007.

[49] B. Azimi-Sadjadi, "Stability of networked control systems in the presence of packet," in Proceedings of the 42nd IEEE Conference on Decision and Control, vol. 1, pp. 676-681, December 2003.

[50] D. Georgiev and D. M. Tilbury, "Packet-based control: the H2optimal solution," Automatica, vol. 42, no. 1, pp. 137-144, 2006.

[51] O. C. Imer, S. Yuksel, and T. Basar, "Optimal control of LTI systems over unreliable communication links," Automatica, vol. 42, no. 9, pp. 1429-1439, 2006.

[52] S. Hu and W.-Y. Yan, "Stability robustness of networked control systems with respect to packet loss," Automatica, vol. 43, no. 7, pp. 1243-1248, 2007.

[53] J. Xiong and J. Lam, "Stabilization of linear systems over networks with bounded packet loss," Automatica, vol. 43, no. 1, pp. 80-87, 2007.

[54] S. Sun, "Linear optimal state and input estimators for networked control systems with multiple packet dropouts," International Journal of Innovative Computing, Information and Control, vol. 8, no. 10, pp. 7289-7305, 2012.

[55] Y.-L. Wang and G.-H. Yang, " $H_{\infty}$ control of networked control systems with time delay and packet disordering," IET Control Theory \& Applications, vol. 1, no. 5, pp. 1344-1354, 2007.

[56] Y.-B. Zhao, J. Kim, G.-P. Liu, and D. Rees, "Compensation and stochastic modeling of discrete-time networked control systems with data packet disorder," International Journal of Control, Automation and Systems, vol. 10, no. 5, pp. 1055-1063, 2012.

[57] Y.-B. Zhao, G.-P. Liu, and D. Rees, "Actively compensating for data packet disorder in networked control systems," IEEE Transactions on Circuits and Systems II: Express Briefs, vol. 57, no. 11, pp. 913-917, 2010. 
[58] H. Zhang, G. Hui, and Y. Wang, "Stabilization of networked control systems with piecewise constant generalized sampleddata hold function," International Journal of Innovative Computing, Information and Control, vol. 9, no. 3, pp. 1159-1170, 2013.

[59] N. Elia and S. K. Mitter, "Stabilization of linear systems with limited information," IEEE Transactions on Automatic Control, vol. 46, no. 9, pp. 1384-1400, 2001.

[60] D. Liberzon, "Quantization, time delays, and nonlinear stabilization," IEEE Transactions on Automatic Control, vol. 51, no. 7, pp. 1190-1195, 2006.

[61] C. Peng and Y.-C. Tian, "Networked $H_{\infty}$ control of linear systems with state quantization," Information Sciences, vol. 177, no. 24, pp. 5763-5774, 2007.

[62] E. Tian, D. Yue, and X. Zhao, "Quantised control design for networked control systems," IET Control Theory and Applications, vol. 1, no. 6, pp. 1693-1699, 2007.

[63] R. Lu, F. Wu, and A. Xue, "Networked control with reset quantized state based on bernoulli processing," IEEE Transactions on Industrial Electronics, vol. 61, no. 9, pp. 4838-4846, 2014.

[64] R. Lu, H. Li, A. Xue, J. Zheng, and Q. She, "Quantized $H_{\infty}$ filtering for different communication channels," Circuits, Systems, and Signal Processing, vol. 31, no. 2, pp. 501-519, 2012.

[65] J.-P. Richard, "Time-delay systems: an overview of some recent advances and open problems," Automatica, vol. 39, no. 10, pp. 1667-1694, 2003.

[66] L. Zhang, Access scheduling and controller design in networked control systems [Ph.D. dissertation], University of Maryland, College Park, Md, USA, 2005.

[67] G. C. Goodwin, D. E. Quevedo, and E. I. Silva, "Architectures and coder design for networked control systems," Automatica, vol. 44, no. 1, pp. 248-257, 2008.

[68] J. Colandairaj, G. W. Irwin, and W. G. Scanlon, "Wireless networked control systems with QoS-based sampling," IET Control Theory and Applications, vol. 1, no. 1, pp. 430-438, 2007.

[69] G.-P. Liu, Y. Xia, D. Rees, and W. Hu, "Design and stability criteria of networked predictive control systems with random network delay in the feedback channel," IEEE Transactions on Systems, Man and Cybernetics Part C: Applications and Reviews, vol. 37, no. 2, pp. 173-184, 2007.

[70] F. Mazenc and S.-I. Niculescu, "Lyapunov stability analysis for nonlinear delay systems," Systems \& Control Letters, vol. 42, no. 4, pp. 245-251, 2001.

[71] H.-J. Hoo, H.-S. Ryu, K.-S. Yoo, and O.-K. Kwon, "Compensation of networked control systems using LMI-based delaydependent optimization method," in Proceedings of the 41st SICE Annual Conference (SICE '02), vol. 1, pp. 364-369, August 2002.

[72] H. Lin, G. Zhai, and P. J. Antsaklis, "Robust stability and disturbance attenuation analysis of a class of networked control systems," in Proceedings of the 42nd IEEE Conference Decision and Control, vol. 2, pp. 1182-1187, Maui, Hawaii, USA, December 2003.

[73] J. K. C. So, Delay modeling and controller design for networked control systems [Ph.D. dissertation], University of Toronto, Toronto, Canada, 2003.

[74] Z.-H. Guan, W.-H. Chen, and J. Xu, "Delay-dependent stability and stabilizability of uncertain jump bilinear stochastic systems with mode-dependent time-delays," International Journal of Systems Science, vol. 36, no. 5, pp. 275-285, 2005.

[75] J. Wu, F.-Q. Deng, and J.-G. Gao, "Modeling and stabilty of long random delay networked control systems," in Proceedings of the 4th International Conference on Machine Learning and
Cybernetics (ICMLC '05), pp. 947-952, Guangzhou, China, August 2005.

[76] L. Zhang and F. Hua-Jing, "A novel controller design and evaluation for networked control systems with time-variant delays," Journal of the Franklin Institute, vol. 343, no. 2, pp. 161$167,2006$.

[77] H. Gao and T. Chen, "New results on stability of discrete-time systems with time-varying state delay," IEEE Transactions on Automatic Control, vol. 52, no. 2, pp. 328-334, 2007.

[78] P. Antsaklis and J. Baillieul, "Guest editorial special issue on networked control systems," IEEE Transactions on Automatic Control, vol. 49, no. 9, pp. 1421-1423, 2004.

[79] M. S. Branicky, S. M. Phillips, and W. Zhang, "Stability of networked control systems: explicit analysis of delay," in Proceedings of the American Control Conference, vol. 4, pp. 23522357, Chicago, Ill, USA, June 2000.

[80] P. Seiler and R. Sengupta, "An $H_{\infty}$ approach to networked control," Institute of Electrical and Electronics Engineers. Transactions on Automatic Control, vol. 50, no. 3, pp. 356-364, 2005.

[81] M. Basin and J. Rodriguez-Gonzalez, "Optimal control for linear systems with multiple time delays in control input," IEEE Transactions on Automatic Control, vol. 51, no. 1, pp. 91-97, 2006.

[82] M. Sahebsara, T. Chen, and S. L. Shah, "Optimal $H_{\infty}$ filtering in networked control systems with multiple packet dropouts," Systems \& Control Letters, vol. 57, no. 9, pp. 696-702, 2008.

[83] G. C. Goodwin, H. Haimovich, D. E. Quevedo, and J. S. Welsh, "A moving horizon approach to networked control system design," IEEE Transactions on Automatic Control, vol. 49, no. 9, pp. 1427-1445, 2004.

[84] Y.-B. Zhao, G.-P. Liu, and D. Rees, "Networked predictive control systems based on the hammerstein model," IEEE Transactions on Circuits and Systems II: Express Briefs, vol. 55, no. 5, pp. 469-473, 2008.

[85] Y.-B. Zhao, G.-P. Liu, and D. Rees, "A predictive controlbased approach to networked hammerstein systems: design and stability analysis," IEEE Transactions on Systems, Man, and Cybernetics, Part B: Cybernetics, vol. 38, no. 3, pp. 700-708, 2008.

[86] Y.-B. Zhao, G.-P. Liu, and D. Rees, "A predictive control based approach to networked wiener systems," International Journal of Innovative Computing, Information and Control, vol. 4, no. 11, pp. 2793-2802, 2008.

[87] N. H. El-Farra and P. D. Christofides, "Coordinating feedback and switching for control of hybrid nonlinear processes," AIChE Journal, vol. 49, no. 8, pp. 2079-2098, 2003.

[88] P. V. Zhivoglyadov and R. H. Middleton, "Networked control design for linear systems," Automatica, vol. 39, no. 4, pp. 743750, 2003.

[89] Z.-H. Guan, D. J. Hill, and X. Shen, "On hybrid impulsive and switching systems and application to nonlinear control," IEEE Transactions on Automatic Control, vol. 50, no. 7, pp. 1058-1062, 2005.

[90] P. A. Kawka and A. G. Alleyne, "Stability and feedback control of wireless networked systems," in Proceedings of the American Control Conference (ACC '05), vol. 4, pp. 2953-2959, Portland, Ore, USA, June 2005.

[91] M. Yu, L. Wang, and G. Xie, "A switched system approach to stabilization of networked control systems," Journal of Control Theory and Applications, vol. 4, no. 1, pp. 86-95, 2006. 
[92] Y. Xia, G. P. Liu, P. Shi, D. Rees, and E. J. C. Thomas, "New stability and stabilization conditions for systems with timedelay," International Journal of Systems Science, vol. 38, no. 1, pp. 17-24, 2007.

[93] X.-M. Sun, D. Wu, G.-P. Liu, and W. Wang, "Input-to-state stability for networked predictive control with random delays in both feedback and forward channels," IEEE Transactions on Industrial Electronics, vol. 61, no. 7, pp. 3519-3526, 2014.

[94] M. S. Branicky, V. Liberatore, and S. M. Phillips, "Networked control system co-simulation for co-design," in Proceedings of the American Control Conference, vol. 4, pp. 3341-3346, 2003.

[95] J. R. Hartman, Networked control system co-simulation for codesign: theory and experiments [Ph.D. dissertation], Department of Electrical Engineering and Computer Science, Case Western Reserve University, Cleveland, Ohio, USA, 2004.

[96] M. E. M. Ben Gaid, A. Çela, and Y. Hamam, "Optimal integrated control and scheduling of networked control systems with communication constraints: Application to a car suspension system," IEEE Transactions on Control Systems Technology, vol. 14, no. 4, pp. 776-787, 2006.

[97] G.-P. Liu, Y. Xia, J. Chen, D. Rees, and W. Hu, "Networked predictive control of systems with random network delays in both forward and feedback channels," IEEE Transactions on Industrial Electronics, vol. 54, no. 3, pp. 1282-1297, 2007.

[98] Y.-B. Zhao, G.-P. Liu, and D. Rees, "Design and stability analysis of packet-based networked control systems in continuous time," in Proceedings of the IEEE International Conference on Systems, Man and Cybernetics (SMC '08), pp. 3206-3210, Singapore, October 2008.

[99] Y.-B. Zhao, G.-P. Liu, and D. Rees, "Packet-based deadband control for internet-based networked control systems," IEEE Transactions on Control Systems Technology, vol. 18, no. 5, pp. 1057-1067, 2010.

[100] Y.-B. Zhao, J. Kim, and G.-P. Liu, "Error bounded sensing for packet-based networked control systems," IEEE Transactions on Industrial Electronics, vol. 58, no. 5, pp. 1980-1989, 2011.

[101] D. Nešić and A. R. Teel, "Input-to-state stability of networked control systems," Automatica, vol. 40, no. 12, pp. 2121-2128, 2004.

[102] M. Tabbara, D. Nešić, and A. Teel, "Stability of wireless and wireline networked control systems," IEEE Transactions on Automatic Control, vol. 52, no. 9, pp. 1615-1630, 2007.

[103] D. Hristu-Varsakelis and P. R. Kumar, "Interrupt-based feedback control over a shared communication medium," in Proceedings of the 41st IEEE Conference on Decision \& Control, vol. 3, pp. 3223-3228, December 2002.

[104] R. Brockett, "Stabilization of motor networks," in Proceedings of the 34th IEEE Conference on Decision and Control, vol. 2, pp. 1484-1488, December 1995.

[105] M. S. Branicky, S. M. Phillips, and W. Zhang, "Scheduling and feedback co-design for networked control systems," in Proceedings of the 41st IEEE Conference on Decision and Control, vol. 2, pp. 1211-1217, Las Vegas, Nev, USA, December 2002. 


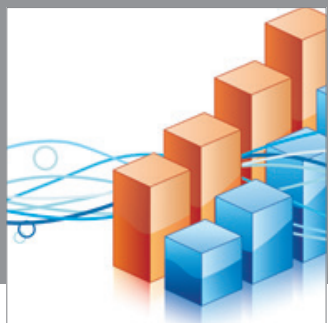

Advances in

Operations Research

mansans

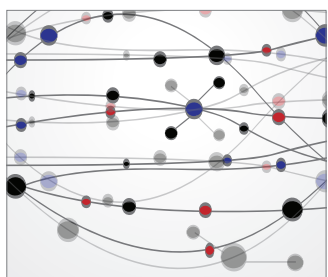

The Scientific World Journal
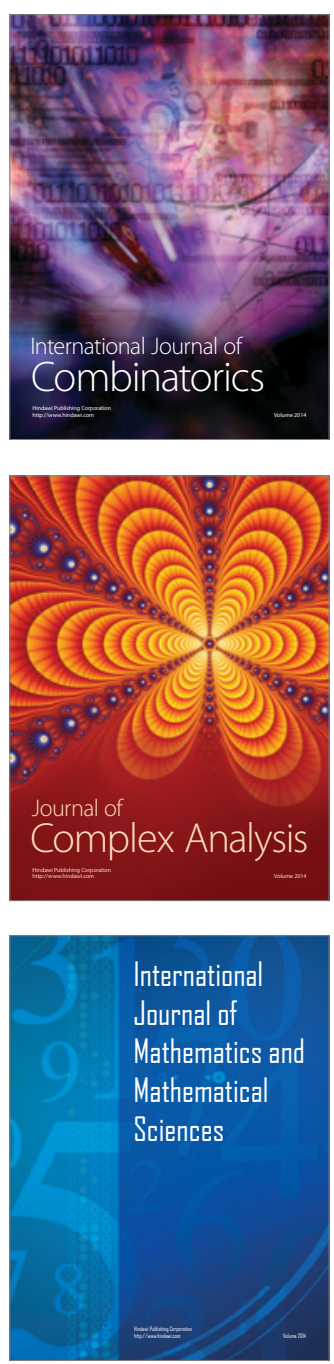
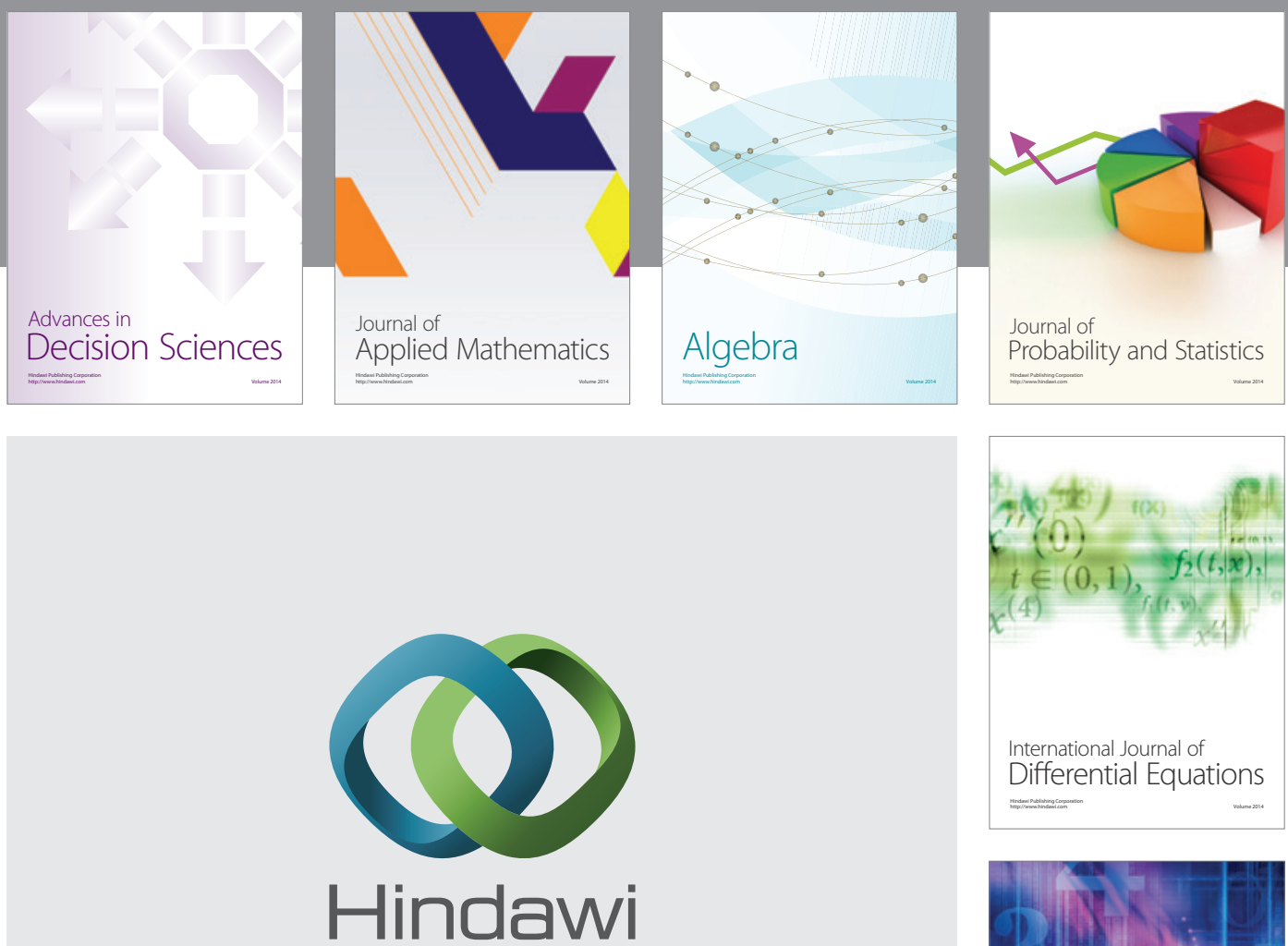

Submit your manuscripts at http://www.hindawi.com
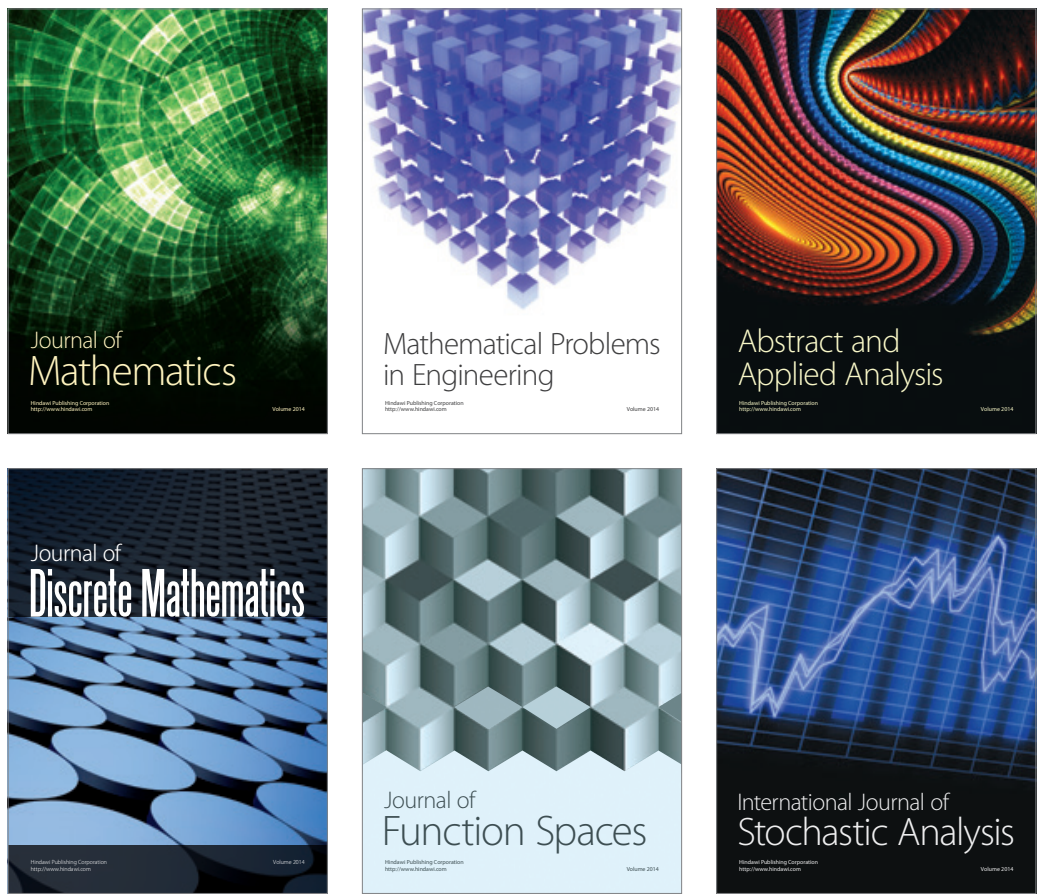

Journal of

Function Spaces

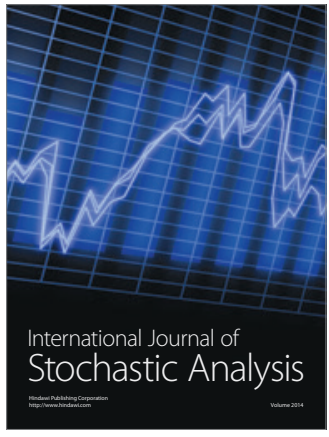

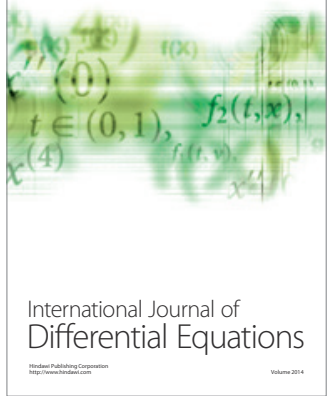
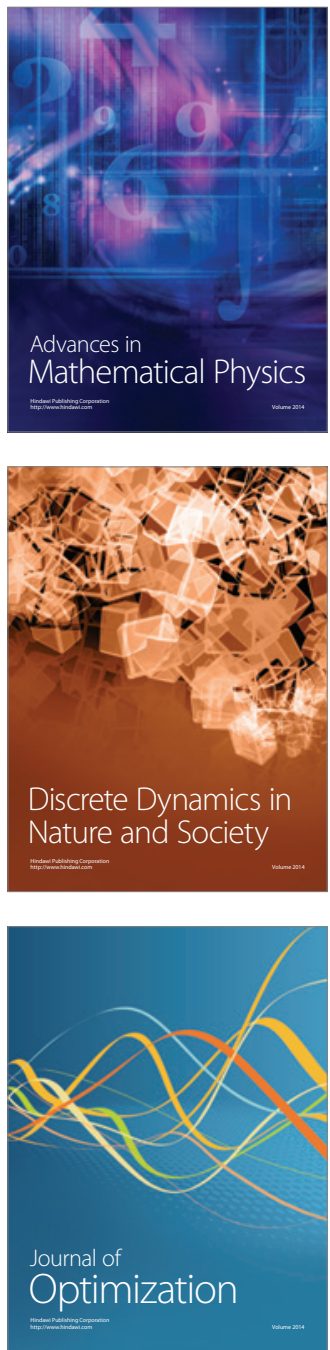